Draft VERSion SEPTEMBER 15, 2020

Typeset using $\mathrm{LAT}_{\mathrm{E}} \mathrm{X}$ modern style in AASTeX61

\title{
THE 2017 SEPTEMBER 6 FLARE: RADIO BURSTS AND PULSATIONS IN THE 22-5000 MHZ RANGE AND ASSOCIATED PHENOMENA
}

\author{
MaRian KARLickÝ ${ }^{1}$ AND JÁN RYBÁK ${ }^{2}$ \\ ${ }^{1}$ Astronomical Institute of the Academy of Sciences of the Czech Republic, CZ-25165 Ondřejov, \\ Czech Republic \\ ${ }^{2}$ Astronomical Institute, Slovak Academy of Sciences, SK-05960 Tatranská Lomnica, Slovakia
}

\begin{abstract}
For the 2017 September 6 flare (SOL2017-Sep-06T11:53) we present not only unusual radio bursts, but also their interesting time association with the other flare phenomena observed in EUV, white-light, X-ray, and $\gamma$-ray emissions. Using our new method based on wavelets we found quasi-periodic pulsations (QPPs) in several locations of the whole time-frequency domain of the analyzed radio spectrum (11:5512:07 UT and 22-5000 MHz). Among them the drifting QPPs are new and the most interesting, especially a bi-directional QPP at the time of the hard X-ray and $\gamma$-ray peaks, and a sunquake start. In the pre-impulsive phase we show an unusual drifting pulsation structure (DPS) in association with the EUV brightenings caused by the interaction of magnetic ropes. In the flare impulsive phase we found an exceptional radio burst drifting from $5000 \mathrm{MHz}$ to $800 \mathrm{MHz}$. In connection with this drifting burst, we show a U-burst at about the onset time of an EUV writhed structure and a drifting radio burst as a signature of a shock wave at high frequencies (1050-1350 $\mathrm{MHz}$ ). In the peak flare phase we found an indication of an additional energy-release process located at higher altitudes in the solar atmosphere. These phenomena are interpreted considering a rising magnetic rope, magnetosonic waves and particle beams. Using a density model we estimated the density, wave velocities and source heights for the bi-directionally drifting QPPs, the density for the pre-impulsive DPS and U-burst, and the density and magnetic field strength for the drifting radio burst.
\end{abstract}

Keywords: Sun: flares - Sun: radio radiation - Sun: oscillations

Corresponding author: Ján Rybák

rybak@astro.sk 


\section{INTRODUCTION}

Radio bursts are an integral part of solar flare observations. These bursts were classified into several types (II, III, V, J, U, and IV) and summarized in several schemes in the time-frequency domain (Krueger 1979; McLean \& Labrum 1985; Pick 1986). They indicate superthermal electrons in solar flares. Many model for their explanation were proposed, see e.g. the books by Melrose (1980); McLean \& Labrum (1985). A catalogue of radio bursts in the 1000-3000 MHz range and their statistics in the 800-2000 MHz range can be found in the papers by Isliker \& Benz (1994); Jiřička et al. (2001).

Quasi-periodic pulsations (QPPs) are also a common feature of solar flares. They were detected in all energy bands, from radio up to $\gamma$-rays. Relations between the QPPs in different bands are of high interest and they were studied in many papers. For example, Aschwanden et al. (1990) showed a weak correlation between decimetric QPPs and hard X-ray emission. On the other hand, a good correlation between the radio on $3 \mathrm{GHz}$, X-ray (11-26 keV), and EUV (18 nm) was found by Karlický et al. (2020b). For an explanation of the QPPs several models were suggested. Aschwanden (1987) classified these models in three groups according to their driving mechanisms: 1) magnetic flux tube oscillations, 2) cyclic self-organizing systems of plasma instabilities, and 3) modulation of particle acceleration. Furthermore, in the paper by Nakariakov \& Melnikov (2009), the authors split the QPP models into two groups: load/unload mechanisms (e.g. the repetitive regimes of flaring energy releases by magnetic reconnection) and magnetohydrodynamic oscillations. For the repetitive regime of the magnetic reconnection, see also the paper by Kliem et al. (2000). A review of magnetohydrodynamic oscillatory processes in the solar corona is presented in Nakariakov et al. (2016). As concerns observational verifications of these models, it looks that the most probable models are those based on quasi-periodic acceleration

and injection of fast electrons (Fleishman et al. 2008; Mohan et al. 2019) or those based on the modulation by magnetohydrodynamic waves (Nakariakov et al. 2006; Carley et al. 2019).

An interesting example of radio QPPs was presented in the paper by Karlický \& Rybák (2017). Here, during the 2010 August 1 flare, QPPs driftingx from 2000 to $400 \mathrm{MHz}$ and with a period of 160-220 s were found. These QPPs were attributed to a fast mode magnetosonic wave train with a $181 \mathrm{~s}$ period propagating upwards in the solar atmosphere (Liu et al. 2011).

In the present paper, based on the analysis of the $22-5000 \mathrm{MHz}$ radio spectrum of the 2017 September 6 flare and in comparison with EUV, white-light, X-ray, and $\gamma$-ray observations, we show several flare induced phenomena not described in literature so far. For example, the burst at the beginning of the flare that drifts from 5000 to $800 \mathrm{MHz}$ (burst B, see the following) differs from the bursts in the radio schemes shown in Krueger (1979); McLean \& Labrum (1985); Pick (1986). Analyzing the radio spectrum we searched for periods and phases of significant quasi-periodic pul- 
sations in the whole $22-5000 \mathrm{MHz}$ radio spectrum using our novel technique based on the wavelet transform (Karlický \& Rybák 2017; Karlický et al. 2017). We found pulsations not only on single frequencies, but in the all time-frequency sub-domains where they are statistically significant. This technique filters the radio spectrum in the selected intervals of time variations and thus enables to show fine features that are on the original spectrum hardly distinguishable. By this way we recognized bidirectionally drifting QPPs that were not found so far. Just the analysis of these drifting QPPs is one of main topics of our paper. We also present interesting time associations between the observed radio bursts and phenomena shown in other papers about this flare. Therefore, our results are complementary to those shown in these papers.

The paper is structured as follows. In Section 2 we present previous observations relevant to our study and describe the $22-5000 \mathrm{MHz}$ radio spectrum of the 2017 September 6 flare. An analysis of the quasi-periodic pulsations in this radio spectrum together with an association of the radio bursts with the phenomena in EUV, whitelight, $\mathrm{X}$ - and $\gamma$-ray observations are given in Section 3. Interpretation and discussion of the presented results are in Section 4 and conclusions are summarized in Section 5 .

\section{OBSERVATIONS}

\subsection{Previous observations}

On 2017 September 6, in the active region NOAA AR12673, the largest flare in Solar Cycle 24, classified as X9.3 flare, was observed. According to the (GOES) observations this flare started at 11:53 UT, reached its peak at 12:02 UT, and ended at 12:10 UT. It was accompanied by a large CME (Yan et al. 2018). This flare was described and analyzed in many papers so far. In one channel of the irradiance measurements the first peak appeared already in the pre-impulsive phase at 11:53:20 UT (Dominique et al. 2018). At the flare location Hou et al. (2018) recognized the double-decker flux rope configuration by the non-linear force free field technique. This configuration erupted as shown by the brightenings starting from 11:53:53 UT and rising writhed structure at 11:56:08 UT. The flare impulsive phase was associated with X-ray up to $\gamma$-ray emissions. The $300-1200 \mathrm{keV}$ time profile showed two peaks: the first stronger one at 11:56-11:56:50 UT and weaker one at 11:57-11:58 UT (Lysenko et al. 2019). During the first peak the analysis revealed contributions from nuclear deexcitation lines, electron - positron annihilation line at $511 \mathrm{keV}$, and a neutron capture line at $2.223 \mathrm{MeV}$ along with two components of the bremsstrahlung continuum (Lysenko et al. 2019). Li et al. (2020) analyzed periods in this flare, in the hard X-rays, $\gamma$-rays and in radio on $1250.9 \mathrm{MHz}$. In the hard X-rays and $1250.9 \mathrm{MHz}$ they found the periods $24-30 \mathrm{~s}$ in the time interval 11:57-11:58 UT, and in the $\gamma$-rays

the period $\sim 20$ s at 11:55:30-11:57 UT. Moreover in this flare, the white-light flare emission and a sunquake were observed (Jurčák et al. 2018; Sharykin \& Kosovichev 
2018). Continuum intensity variations were detected close to double-decker flux rope configuration starting at 11:54:49 UT and this location was found as that where the helioseismic wave started (Sharykin \& Kosovichev 2018).

\subsection{Data}

For preparation of the broadband $22-5000 \mathrm{MHz}$ radio spectrum we used data from four different radiospectrographs: Greenland-Callisto radiospectrograph working in the 22-100 MHz range with the resolutions of $0.25 \mathrm{~s}$ and $0.48 \mathrm{MHz}$ (Monstein 2016), ORFEES radiospectrograph working in the $150-1000 \mathrm{MHz}$ range with the resolutions of $1.0 \mathrm{~s}$ and $0.98 \mathrm{MHz}$ (publicly available data, Observation Radio Frequence pour l'Etude des Eruptions Solaires radiospectrograph, Nancay, France), and the Ondřejov radiospectrographs working in the ranges $800-2000 \mathrm{MHz}$ and $2000-5000 \mathrm{MHz}$ with the resolutions of $0.01 \mathrm{~s}$ and $4.7 \mathrm{MHz}$, and $0.01 \mathrm{~s}$ and $11.7 \mathrm{MHz}$ (Jiřička \& Karlický 2008), respectively. At some frequencies, human-made radio interference affected the acquired signal. Such parts of the data are excluded and they are marked by dark bands in all plots of the radiospectrograms. Some parts of the spectra were saturated due to very strong signals. However, the presented results are not influenced by these saturated data.

The final frequency range $22-5000 \mathrm{MHz}$ range is complete except a minor $100-150$ $\mathrm{MHz}$ gap and the sub-range $800-1000 \mathrm{MHz}$ is duplicated. The final time-frequency radiospectrum was chosen in the time interval 11:55-12:07 UT (Figure 3). Because the 2000-5000 MHz Ondřejov radiospectrograph registered also a weak radio emission at about 11:53:54-11:55:00 UT, i.e., before the start of all other bursts in the 22-5000 $\mathrm{MHz}$ range, these data were also analyzed.

We also used soft X-ray fluxes observed by the GOES 15 satellite analyzing the flux data of $2 \mathrm{~s}$ cadence for this time interval. Moreover, we evaluated photometrically reduced EUV images from the SDO/AIA instrument (Lemen et al. 2012) in several channels with the AIA stray-light point-spread function correction applied (Poduval et al. 2013).

\subsection{Description of radio data}

In Figure 1 the time profiles of the radio flux on 65, 550, 1050 and $2800 \mathrm{MHz}$ together with GOES 15 0.5-4 $\AA$ and 1-8 $\AA$ X-ray fluxes during the 2017 September 6 event are shown. Owing to very strong radio fluxes during this flare some parts of these profiles are saturated. As seen at $2800 \mathrm{MHz}$ and GOES time profiles, the flare started at about 11:54 UT and its maximum in the radio emission drifts from high to lower frequencies. To understand better the evolution of the radio emission during this flare, its radio spectra are presented in Figures 2 and 3.

Figure 2 shows a remarkable drifting pulsation structure (DPS) that appears on radio waves in the $2200-4200 \mathrm{MHz}$ range just before the main radio event displayed in Figure 3. It looks that this DPS consists of two parts. The part 1 starts at 11:53:54 UT and ends at 11:55:00 UT. The part 2 is stronger and starts at 11:54:18 UT. It 
is superposed on the first one and it drifts from higher to lower frequencies with the mean frequency drift of about $-20 \mathrm{MHz} \mathrm{s}^{-1}$. This double DPS is unusual not only that consists of two parts, but also because it is observed already in the pre-impulsive flare phase and at high-frequencies. Usually DPSs are observed in the 1-2 GHz range and they are signatures of the flare impulsive phase (Nishizuka et al. 2015).

Figure 3 shows a global overview of radio bursts in the $22-5000 \mathrm{MHz}$ range in the time interval 11:55-12:07 UT. There are strong pulsations $(\mathrm{P})$ at its beginning at 11:55:20-11:57:00 UT in the 1800-4000 MHz range, see also the details of these quasi-periodic pulsations in panels $\mathrm{d}$ and e in Figure 7 . With some time delay, at about 11:56 UT, the burst (designated as B) starts at $5000 \mathrm{MHz}$ and drifts to 800 $\mathrm{MHz}$. It reaches the frequency $800 \mathrm{MHz}$ at about 11:58:30 UT. The mean frequency drift of this burst $\mathrm{B}$ is about $-28 \mathrm{MHz} \mathrm{s}^{-1}$. In the $150-2000 \mathrm{MHz}$ range this burst is followed by the type IV radio burst. We note that the burst B is exceptional, mainly due to its relatively low frequency drift and its form of the emission band lasting about two minutes and drifting as a whole from $5000 \mathrm{MHz}$ to $800 \mathrm{MHz}$. Namely, in this frequency range and in this flare phase we usually observe broadband continua without any frequency drift or fast drifting bursts having the frequency drift of about $\pm 1 \mathrm{GHz} \mathrm{s}^{-1}$ (Jiřička et al. 2001). The burst B differs from the flare radio bursts in the radio schemes shown in Krueger (1979); McLean \& Labrum (1985); Pick (1986) and also from the bursts shown, e.g., in broadband radio spectra in the papers by Pick et al. (2005); Bain et al. (2014). During more than 30 years of observations by the Ondřejov radiospectrographs we found only three examples with the radio bursts that were partly similar to the present burst: in 1992 February 27, X3.3 flare (Karlický \& Odstrčil 1994), in 1996 July 9, X2.6 flare (Karlický 1998), and in 2000 July 12, X1.9 flare (Karlický et al. 2001). On lower frequencies this burst B is followed by the type II radio burst at 12:03-12:07 UT in the 22-100 $\mathrm{MHz}$ range. Before the type II burst, at 11:57-12:03 UT in the 22-100 MHz range, type III bursts can be seen. Moreover, QPPs are omnipresent in the radio spectrum, see e.g. Figure 6.

The $22-5000 \mathrm{MHz}$ radio spectrum is rich in fine structures. The most remarkable fine structures are shown in Figure 4. In the panel a of this figure, in the 1050-1300 $\mathrm{MHz}$ range at about 11:55:52-11:56:00 UT, a group of bursts starting with the highfrequency type U-burst (U) is presented. In the panel b, in the 1050-1350 MHz range and at 11:57:19-11:57:37 UT, just before the onset boundary of the drifting burst B, we found an unusual drifting radio burst (DRB) consisting of many spikes.

\section{RADIO SPECTRUM ANALYSIS}

\subsection{Periods and phases of radio pulsations}

As mentioned above, pulsations are omnipresent in the radiospectrum of this flare. Therefore we searched for any periodic signal of these pulsations in the whole radio spectrum. We used the same technique as presented in Karlický \& Rybák (2017) and Karlický et al. (2017). It is based on the wavelet transform (WT) (Torrence \& Compo 
1998) providing a clear detection of time-frequency evolution of the strong radio wave-patterns. Before calculations the Ondřejov radiospectrum data were resampled to $0.25 \mathrm{~s}$ temporal resolution. No other special data reduction has been applied to the data of radiospectrographs except a resampling of the ORFEES data to an equidistant frequency scale. The Morlet mother wavelet, consisting of a complex sine wave modulated by a Gaussian, was selected to search for radio signal variability, with the non-dimensional frequency $\omega_{0}$ satisfying the admissibility condition (Farge 1992). The WT was calculated for the period range starting from 4 time steps with scales sampling the signal sufficiently as fractional power of two with $\delta j=0.4$ (see Eq. 9. Torrence \& Compo (1998)). Both the calculated significance of the derived WT periodicities and the cone-of-influence were taken into account as described in Karlický \& Rybák (2017). The value of the confidence level was set to $99 \%$.

First, we computed the histograms of the detected periods in all four radio spectra. The results are shown in Figure 5. Each peak in these histograms represents a group of significant periodic signals of a roughly similar period. As seen here, the lower frequency of observations results in a detection of longer periods.

To show temporal and frequency location of these periods, for each peak in the period histograms we made maps of the period phases which we overplotted on the radio spectra. Because of too many such maps, we present here only examples of the most interesting maps (Figure 6). In the 22-100 MHz range the phases fully cover the original radio spectrum, therefore the $22-100 \mathrm{MHz}$ original spectrum is added (Figure 6a1). For other maps the original spectra are much better visible and they can be also seen in Figure 3. We found that most of the pulsations are located at the same positions in the time-frequency domain. It indicates multi-periodic processes at these locations in the time-frequency domain. These multi-periodic processes were found at 11:55:20-11:57 UT in the 1800-4000 MHz range, at 11:59-12:00:25 UT in the 800$2000 \mathrm{MHz}$ range, at 11:57:30-11:58:20 UT and 11:59:30-12:01 UT in the 150-1000 $\mathrm{MHz}$ range, and at 11:57-12:02 $\mathrm{UT}$ in the $22-100 \mathrm{MHz}$ range.

The most interesting time interval with pulsations is that of the first $\gamma$-ray peak at 11:56-11:56:50 UT (Lysenko et al. 2019). Therefore, in Figure 7 we present three detailed phase maps for the radio frequency bands $2000-5000 \mathrm{MHz}$ for the periods 1.0 $2.0 \mathrm{~s}, 5.3-8.5 \mathrm{~s}$, and $11-30 \mathrm{~s}$ together with the original spectrum and its frequency cuts. As seen here, the phase of pulsations at 11:55:50-11:56:40 UT with periods $1.0-2.0 \mathrm{~s}$ is synchronized in the whole frequency range for these periods (black lines that designate the zero phase are vertical (Figure 7, panel a)). On the other hand, at about 11:56-11:56:30 UT the pulsations with the periods $5.3-8.5 \mathrm{~s}$ and $11-30 \mathrm{~s}$ show the frequency drift (Figure 7, panels b and c): the negative drift of about $170 \mathrm{MHz} \mathrm{s}^{-1}$ for frequencies below $3000 \mathrm{MHz}$ and the positive drift of about 330 $\mathrm{MHz} \mathrm{s}^{-1}$ for frequencies above $3500 \mathrm{MHz}$. Furthermore, in Figure 8 we show similar oppositely drifting pulsations for periods $15-20 \mathrm{~s}$ at 11:59:10-11:59:40 UT in the 800- 
$1000 \mathrm{MHz}$ range (D1) and at 11:59:50-12:00:10 UT in the 350-450 MHz range (D2). The frequency drift is $30 \mathrm{MHz} \mathrm{s}^{-1}$ and $-8 \mathrm{MHz} \mathrm{s}^{-1}$, respectively.

3.2. Association of radio bursts with EUV, white-light, X-ray, and $\gamma$-ray observations

We found several interesting time associations between radio bursts and their fine structures with the flare phenomena observed in EUV, white-light, X- and $\gamma$-rays:

a) The initial double DPS was observed at 11:53:54 UT-11:55:00 UT. At this time interval, in the He II $304 \AA$ images (50 kK, Lemen et al. (2012)) several brightenings were observed: at north cross site at 11:53:53 UT, at south end of Flux rope 2 at 11:54:29 UT, at south end of Flux rope 1 at 11:54:41 UT, and at south cross site at 11:54:53 UT (see Figure 3 in Hou et al. (2018) and Figure 9 bottom panel). These brightenings were described as caused by interactions between Flux rope 1 and Flux rope 2 (Hou et al. 2018). Furthermore, at about the same time (at 11:53:20 UT) the first small peak in LYRA Channel 2 (irradiance measurement in the 1900-2220 $\AA$ range) was detected (Dominique et al. 2018).

b) At the time interval 11:55:20-11:57:00 UT in the 1800-4000 MHz range (Figure 7) the 1-30 s pulsations with either infinite or with finite and oppositely drifting phases (see previous sub-section) were observed simultaneously. For this time interval the hard X-ray $(83-331 \mathrm{keV})$ and $\gamma$-ray $(331-1253 \mathrm{keV})$ peaks with contributions from nuclear deexcitation lines, electron-positron annihilation line at $511 \mathrm{keV}$, and a neutron capture line at $2.223 \mathrm{MeV}$ along with two components of the bremsstrahlung continuum were observed (Lysenko et al. 2019). At this time also the sunquake (Sharykin \& Kosovichev 2018) and white-light flare (Jurčák et al. 2018) started, and oscillations in the $\gamma$-ray emission with the period $\sim 20$ s were detected (Li et al. 2020).

c) The burst B in Figure 3 started at about 11:56 UT on $5000 \mathrm{MHz}$ and drifted to lower frequencies down to $800 \mathrm{MHz}$. Its start coincided with the start of the upward motion of magnetic ropes at 11:55:53 UT, as can be seen in Figure 3(a6) in Hou et al. (2018).

d) Moreover, at the time interval 11:55:52-11:56:00 UT in the 1000-1300 MHz range, we found a group of bursts starting with the high-frequency type U-burst. With some short time delay at 11:56:08 UT the EUV writhed structure was observed as it was documented by Hou et al. (2018).

\section{INTERPRETATION AND DISCUSSION}

We have no information about radio bursts spatial positions. Therefore, in the following interpretations drawn below, we rely on the results derived from the spatially resolved observations, already presented in the published articles about this flare as well as on general knowledge about similar phenomena.

The main topics of the paper are the drifting bursts and the drifting QPPs. Although we cannot exclude for them the models based, e.g., on the electroncyclotron maser mechanism (Carley et al. 2019) or the modulated gyro-synchrotron 
emission mechanism (Fleishman et al. 2008), for the drifting phenomena (especially bi-directional) the models based on the plasma emission mechanism are the most probable (Melrose 1980). Therefore, in the following interpretation we will use the models based on the plasma emission mechanism.

In this case the time-frequency information from the radio spectrum about the drifting phenomena means the time-height (in solar atmosphere) information about these bursts. Thus, the frequency drift of the drifting bursts and the drifting QPPs can be interpreted by motion of agents (particle beams or waves) in the vertical direction in the gravitationally stratified solar atmosphere.

For this purpose, we need to use some models of the solar atmosphere. There are several models, e.g., models by Allen (1947); Newkirk (1961); Maxwell \& Thompson (1962); Palmer (1974); Mann et al. (1999); Aschwanden (2002). However, only the model of Aschwanden (2002) corresponds to high plasma frequencies, i.e. to low atmospheric heights, that are of our interest in this paper. Moreover, this model was derived from the radio observations. That is why we will use the model of Aschwanden (2002). However, note that any estimations made by this model are dependent on this model.

The plasma emission mechanism is a two-steps mechanism (Melrose 1980). Firstly, some agent generates the local plasma (Langmuir) waves and then these waves are transformed by non-linear processes to the electromagnetic (radio) waves. The frequency of the radio waves corresponds to the local plasma frequency (fundamental emission branch) or double plasma frequency (harmonic emission branch). The intensity of the emission in the emission source on the fundamental frequency is usually stronger than that on the harmonic frequency. On the other hand, the emission on the fundamental frequency is more strongly absorbed than that on the harmonic frequency, except the case with the fibrous medium (ducting propagation) (Benz 1993). Because in the emission source the medium can be fibrous and because we have no additional information (e.g. polarization of these bursts), we are not able to recognize if the emission is on the fundamental or harmonic frequency. Therefore, the following estimations will be presented in the both variants. We note that in some models of the radio bursts based on the plasma emission mechanism the upper-hybrid waves are considered instead of the Langmuir waves, see e.g. Spicer et al. (1982). In the following, if not expressed explicitly we use the plasma emission mechanism with the Langmuir waves.

In the pre-impulsive phase of the flare, in the $2200-4200 \mathrm{MHz}$ frequency range, we present the unusual drifting pulsation structure (DPS). It consists from two parts. Usually DPSs are observed in the impulsive phase and in the 1000-2000 MHz range (Nishizuka et al. 2015). They are interpreted as the radio emission of superthermal electrons trapped in a plasmoid (Kliem et al. 2000). In the present case, the DPS was observed at time of interactions of two magnetic ropes (Hou et al. 2018). This type of the interaction between ropes is known; it heats the ropes and accelerates 
particles (Sakai \& de Jager 1996). To confirm the heating and acceleration processes in the rope interaction region at the DPS time, in Figure 9 we show the time profiles on radio frequencies 2700, 3000 and $3300 \mathrm{MHz}$ (frequency cuts of the DPS), the GOES soft X-ray fluxes with their time derivatives, and the AIA 304, 335 and 1700 $\AA$ emission fluxes from the rope interaction region. Comparing the profiles of the time derivative of the GOES flux (panel c) with the DPS radio profiles (panel a) and the DPS radio spectrum in Figure 2, it can be seen that the small enhancement in the GOES time derivative at 11:54:03 - 11:54:07 UT (1 in panel c) roughly corresponds to the weaker part of the DPS (Part 1). On the other hand, the stronger enhancement in the GOES time derivative at 11:54:24 - 11:54:30 UT (2 in panel c) together with those in AIA profiles roughly correspond to the stronger part of the DPS (Part 2). While the enhancements in the AIA emission fluxes from the rope interaction region indicate the plasma heating, the enhancements in the GOES derivatives show a presence of the superthermal electrons as follows from the Neupert effect (Neupert 1968; Dennis \& Zarro 1993). These superthermal electrons can be then trapped in these interacting ropes and generate by the plasma emission mechanism two DPSs (Part 1 and Part 2 in Figure 2). In this interpretation the similar frequency band of the both parts of DPS means the similar plasma density in these interacting ropes: $5.9 \times 10^{10}$ $-2.2 \times 10^{11} \mathrm{~cm}^{-3}$ (fundamental emission) or $1.5 \times 10^{10}-5.4 \times 10^{10} \mathrm{~cm}^{-3}$ (harmonic emission). This double DPS resembles to the double structure of the DPS in the 2017 September 10 flare (Karlický et al. 2020a), where the plasma density in the magnetic rope and flare arcade were different and thus two isolated DPS structures were seen in the radio spectrum.

In the impulsive phase, a negatively drifting burst from $5000 \mathrm{MHz}$ to $800 \mathrm{MHz}$ was found (burst B in Figure 3). As shown above this burst is exceptional. In agreement with EUV observations (Hou et al. 2018) we interpret this burst as generated by the rising magnetic rope that accelerates superthermal electrons along its trajectory. Then these electrons generate the drifting burst by the plasma emission mechanism. In such an interpretation the frequency drift of this burst corresponds to the velocity of the rising rope. In the Aschwanden's model of the solar atmosphere, for the drifting burst $\mathrm{B}$ the mean velocity is about $310 \mathrm{~km} \mathrm{~s}^{-1}$ (fundamental emission) or $560 \mathrm{~km} \mathrm{~s}^{-1}$ (harmonic emission), which roughly corresponds to the velocities of rope and loops in the image plane $\left(\sim 200-380 \mathrm{~km} \mathrm{~s}^{-1}\right)$ shown in Figure 5 in the paper by Hou et al. (2018).

Just before and during this burst (burst B), at the time interval 11:55:20-11:57:00 UT in the 1800-4000 MHz we recognized pulsations. At this time the $\gamma$-ray emission together with the white-light flare (Jurčák et al. 2018) and start of the sunquake (Sharykin \& Kosovichev 2018) were detected. These radio pulsations were multiperiodic. The pulsations of the shortest periods (1-2 s) were without measurable frequency drift. On the other hand, the pulsations with the periods 5.3-8.5 s and 11-30 s show the bi-directional drift: the negative frequency drift for the frequencies 
below $3000 \mathrm{MHz}$ and the positive drift above $3500 \mathrm{MHz}$. The frequency drift of these bi-directional QPPs is much smaller than the drift of type III bursts, generated by particle beams, in this frequency range. Namely, assuming the Aschwanden's density model, the frequency drifts of these pulsations correspond to the velocities $880 \mathrm{~km}$ $\mathrm{s}^{-1}$ downwards and $1070 \mathrm{~km} \mathrm{~s}^{-1}$ upwards (fundamental emission) or $1570 \mathrm{~km} \mathrm{~s}^{-1}$ downwards and $1900 \mathrm{~km} \mathrm{~s}^{-1}$ upwards (harmonic emission) in the solar atmosphere. We note that these velocities are only those in the direction of the density gradient and the real velocities of the agents generating these QPPs can be higher. Considering these velocities and the QPP detected in the radio spectrum of the 2010 August 1 flare (Karlický \& Rybák 2017), where the fast mode magnetosonic wave was observed (Liu et al. 2011) we propose that the present bi-directional QPPs are generated by modulation of the radio emission by fast mode magnetosonic waves. They propagate upwards and downwards from their source. Thus, their source should be at the altitude in the solar atmosphere where the plasma frequency is $3000-3500 \mathrm{MHz}$. In the density model of the solar atmosphere, the corresponding source altitude and plasma density is $13800-15700 \mathrm{~km}$ and $1.1 \times 10^{11}-1.5 \times 10^{11} \mathrm{~cm}^{-3}$ (fundamental emission) or 24800-28200 km and $2.8 \times 10^{10}-3.8 \times 10^{10} \mathrm{~cm}^{-3}$ (harmonic emission). Because the quasi-periodic magnetic reconnection is a natural source of fast mode magnetosonic waves (Jelínek et al. 2017) we think that the source of the present magnetosonic waves is the magnetic reconnection. On the other hand, the pulsations with the periods 1-2 s and with no measurable drift can be generated by the quasiperiodic magnetic reconnection (Kliem et al. 2000) or by the electron-cyclotron maser mechanism (Aschwanden \& Benz 1988). Thus, it is possible that all these QPPs (with the periods $1-2 \mathrm{~s}, 5.3-8.5 \mathrm{~s}$ and $11-30 \mathrm{~s}$ ) are generated by a single process, i.e. by the quasi-periodic magnetic reconnection.

We found a time association between a group of bursts starting with the U-burst in the 1050-1300 MHz range with the EUV writhed structure (Hou et al. 2018). We note that non-thermal (radio) processes usually precede the thermal (heating) ones, what could explain the short delay between the EUV writhed-structure observations and the preceding in time radio bursts. According to the theory, the U-burst is generated by the electron beam propagating along a closed loop, see e.g. Aschwanden (2004). The EUV writhed-structure looks to be a kinked magnetic rope. The kinked magnetic rope is not only a closed loop, but in this structure electrons can be accelerated due to the magnetic reconnection between kinked magnetic field lines; for the modeled and observed kinked magnetic rope see also the papers by Kliem et al. (2010); Karlický \& Kliem (2010). Thus, the conditions for the U-burst generation (closed loop and electron acceleration) are fulfilled in this structure. No other Ubursts were observed. If we assume that the U-burst is generated by the electron beam, propagating along this structure, through the plasma emission mechanism, then from the turning (lowest) frequency of the U-burst we can estimate the density 
at the top of the writhed structure as $1.36 \times 10^{10} \mathrm{~cm}^{-3}$ (fundamental emission) or $3.1 \times 10^{9} \mathrm{~cm}^{-3}$ (harmonic emission).

The unusual drifting radio burst (DRB) was detected just before the onset boundary of the drifting burst B at 11:57:19-11:57:37 UT in the 1050-1350 MHz range (DRB in Figure 4b). Its frequency drift is similar to that of the burst B. Thus, the velocity of the possible agent is also similar to those of the burst B: $340 \mathrm{~km} \mathrm{~s}^{-1}$ (fundamental emission) or $620 \mathrm{~km} \mathrm{~s}^{-1}$ (harmonic emission). (Note that these velocities are the velocities in the density gradient direction and real velocities can be higher.) The DRB resembles to the drifting chains of type I observed in the metric frequency range (Elgarøy 1977). The most promising model of these chains is the model by Spicer et al. (1982), explaining the type-I chains by weakly super-Alfvenic shocks generated in the front of emerging magnetic flux. In agreement with this interpretation and similar frequency drift of the DRB and the burst B we propose that the DRB is generated by the rising magnetic rope. The magnetic field around the rising rope is structured, thus some part of the rising rope can propagate through a region with a relatively lower magnetic field strength. In this region the velocity of the rising rope overcomes the Alfvén velocity and a weak shock is formed. At this shock the Langmuir waves (as in the type II burst) or upper-hybrid waves (Spicer et al. 1982) are generated and after their transformation into the radio waves the DRB is produced. This interpretation enables to determine not only the plasma density, but also the magnetic field strength. Assuming the weakly super-Alfvenic shock (Alfvén Mach number $\sim 1$ ) and the plasma emission mechanism with the Langmuir waves then the mean plasma density and the magnetic field strength at the DRB source is $1.8 \times 10^{10} \mathrm{~cm}^{-3}$ and $22.3 \mathrm{G}$ (fundamental emission) or $4.4 \times 10^{9} \mathrm{~cm}^{-3}$ and 20.4 $\mathrm{G}$ (harmonic emission). In the case of the DRB generation by the plasma emission mechanism with the upper-hybrid waves (Spicer et al. 1982), the upper-hybrid waves are generated by the double-plasma resonance instability (Zheleznyakov \& Zlotnik 1975; Benáček \& Karlický 2018) under the condition $s \omega_{c e} \approx \omega_{u h} \approx\left(\omega_{p e}^{2}+\omega_{c e}^{2}\right)^{1 / 2}$, where $\omega_{u h}, \omega_{p e}$, and $\omega_{c e}$ are the upper-hybrid, electron plasma and electron-cyclotron frequencies, respectively, and $s$ is gyro-harmonic number. This gyro-harmonic number is usually much greater than 1 . In such a case the upper-hybrid frequency is close to the plasma (Langmuir wave) frequency. Thus, the estimated plasma density and magnetic field in the model with the upper-hybrid waves are close to those estimated above. The height of the DRB source, in the solar atmosphere, in the Aschwanden's density model is 31000-38000 km (fundamental emission) and 55000-68000 (harmonic emission). Now, using the relation for the model magnetic field in the solar atmosphere $B(R)=0.5\left(R / R_{\odot}-1\right)^{-1.5} \mathrm{G}$ (where $R=R_{\odot}+h, R_{\odot}$ is the solar radius and $h$ is the height in the solar atmosphere) (Dulk \& McLean 1978), the magnetic field strength at the heights $31000-38000 \mathrm{~km}$ is 53-39 G and at the heights 55000-68000 $\mathrm{km}$ is $22-16 \mathrm{G}$. As seen here, the estimated magnetic field for the harmonic emission is in a better agreement with the model magnetic field than that for the fundamental 
emission. But, it does not exclude that the emission of the DRB is on the fundamental frequency, because the real values of the magnetic field in the DRB source region, where the weak super-Alfvenic shock is formed, can be lower than the mean model magnetic field.

Furthermore, in the time interval 11:59-12:01 UT in the 350-400 MHz and 800-1000 $\mathrm{MHz}$ ranges we found the pulsations of the $15-20 \mathrm{~s}$ period with the bi-directional drifts (D1 and D2 in Figure 8). The drift was $30 \mathrm{MHz} \mathrm{s}^{-1}$ for D1 and $-8 \mathrm{MHz}$ $\mathrm{s}^{-1}$ for D2. In the density model of the solar atmosphere (Aschwanden 2002) these frequency drifts correspond to the velocity $1360 \mathrm{~km} \mathrm{~s}^{-1}$ downwards and $1690 \mathrm{~km} \mathrm{~s}^{-1}$ upwards (fundamental emission) or $2400 \mathrm{~km} \mathrm{~s}^{-1}$ downwards and $3000 \mathrm{~km} \mathrm{~s}^{-1}$ upwards (harmonic emission) in the solar atmosphere. Such velocities look like velocities of magnetosonic waves. Because they are bi-directional we expect that these waves were initiated in the source located at the plasma level with the plasma frequency in the 400-800 MHz range, i.e., with the plasma densities $2.0 \times 10^{9}-7.9 \times 10^{9} \mathrm{~cm}^{-3}$ (fundamental emission) or $4.9 \times 10^{8}-2.0 \times 10^{9} \mathrm{~cm}^{-3}$ (harmonic emission). In the density model this frequency range corresponds to the height range $47800-85700 \mathrm{~km}$ (fundamental emission) or $85700-153000 \mathrm{~km}$ (harmonic emission). Comparing this height range with that of the source of the pulsations at 11:56-11:56:30 UT (see Figure 7), we can see that the energy-release source at 11:59-12:01 UT is at higher altitudes than that at 11:56-11:56:30 UT.

As concerns the quasi-periodic pulsations with the period 53-100 s found in the 22-100 MHz range, we think that they are related to type III bursts because they have similar frequency drift as type III bursts in this frequency range. This is clearly seen comparing panels a1 and a2 of Figures 6. These QPPs indicate a presence of some continuum formed from many weak type III bursts.

\section{CONCLUSIONS}

In the paper we present not only some unusual radio bursts and fine structures, but also their interesting time association with the phenomena observed in EUV, whitelight, X-ray and $\gamma$-ray emissions of the 2017 September 6 flare. Furthermore, we show significant quasi-periodic pulsations (the periods and phases) in the time-frequency domain of the analyzed radio spectrum (11:55-12:07 UT and 22-5000 MHz). Especially, the bi-directional QPPs with the positive and negative frequency drifts are the most remarkable. We note that the detection of these drifting QPPs were enabled by our new method for computation of periods and their phases in radio spectra.

We found the double DPS in the pre-impulsive flare phase coupled with the EUV brightenings caused by an interaction of two magnetic ropes. Quasi-periodic pulsations at the beginning of the impulsive flare phase, when the hard X-and $\gamma$-ray emission, white-light flare and sunquake started, were multi-periodic. While phases of the short periods $(1-2 \mathrm{~s})$ have the infinite frequency drift, the longer periods (5.3-8.5 
and 11-30 s) showed the oppositely drifting phases. These bi-directional QPPs were interpreted as caused by the magnetosonic waves.

Furthermore, we presented a group of bursts starting with the U-burst at about the time of the EUV writhed structure and the unusual burst B drifting from 5000 to 800 $\mathrm{MHz}$, which we interpreted as caused by the rising magnetic rope. In front of this burst we found the drifting radio burst (DRB) which we proposed to be a signature of the weakly super-Alfvenic shock, observed on unusually high frequencies (1050-1350 $\mathrm{MHz}$ ) and generated by the rising magnetic rope.

Considering the plasma emission mechanism for all analyzed drifting bursts and using the density model of the solar atmosphere we estimated the density, wave velocities and source heights for the bi-directionally drifting QPPs, density for the preimpulsive DPS and U-burst, and density and magnetic field for the drifting structure. We showed that the energy-release process (magnetic reconnection) moved upwards in the solar atmosphere during the flare.

The authors thank the referee for useful comments that improved the paper. We acknowledge support from the project RVO:67985815 and GA ČR grants 18-09072S, 19-09489S, 20-09922J, and 20-07908S. This work was also supported by the Science Grant Agency project VEGA 2/0048/20 (Slovakia). Help of the Bilateral Mobility Projects SAV-18-01 of the SAS and CAS is acknowledged. This article was created in the project ITMS No. 26220120029, based on the supporting operational Research and development program financed from the European Regional Development Fund. The authors are indebted to the Institute for Particle Physics and Astrophysics, ETH Zurich and FHNW Brugg/Windisch (Switzerland) for the Callisto data as well as the Paris Observatory for the ORFEES data. This research has used of NASA Astrophysics Data System. The wavelet analysis was performed with the software based on tools provided by C. Torrence and G.P. Compo at http://paos. colorado.edu/research/wavelets.

\section{REFERENCES}

Allen, C. W. 1947, MNRAS, 107, 426

Aschwanden, M. J. 1987, SoPh, 111, 113

-. 2002, SSRv, 101, 1

-. 2004, Physics of the Solar Corona. An Introduction, Springer, Praxis Publ., Chichester, UK, p. 658

Aschwanden, M. J., \& Benz, A. O. 1988, ApJ, 332, 466

Aschwanden, M. J., Benz, A. O., \& Kane, S. R. 1990, A\&A, 229, 206

Bain, H. M., Krucker, S., Saint-Hilaire, P., \& Raftery, C. L. 2014, ApJ, 782, 43
Benáček, J., \& Karlický, M. 2018, A\&A, 611, A60

Benz, A. O. 1993, Plasma astrophysics: Kinetic processes in solar and stellar coronae, Kluwer Academic Publ. Dordrecht, The Netherlands, p. 277, Vol. 184, doi:10.1007/978-94-011-2064-7

Carley, E. P., Hayes, L. A., Murray, S. A., et al. 2019, Nature Communications, 10, 2276

Dennis, B. R., \& Zarro, D. M. 1993, SoPh, 146, 177 
Dominique, M., Zhukov, A. N., Heinzel, P., et al. 2018, ApJL, 867, L24

Dulk, G. A., \& McLean, D. J. 1978, SoPh, 57, 279

Elgarøy, E. Ø. 1977, Solar noise storms, Pergamon Press, Oxford, UK

Farge, M. 1992, Annual Review of Fluid Mechanics, 24, 395

Fleishman, G. D., Bastian, T. S., \& Gary, D. E. 2008, ApJ, 684, 1433

Hou, Y. J., Zhang, J., Li, T., Yang, S. H., \& Li, X. H. 2018, A\&A, 619, A100

Isliker, H., \& Benz, A. O. 1994, A\&AS, 104, 145

Jelínek, P., Karlický, M., Van Doorsselaere, T., \& Bárta, M. 2017, ApJ, 847, 98

Jiřička, K., \& Karlický, M. 2008, SoPh, 253, 95

Jiřička, K., Karlický, M., Mészárosová, H., \& Snížek, V. 2001, A\&A, 375, 243

Jurčák, J., Kašparová, J., Švanda, M., \& Kleint, L. 2018, A\&A, 620, A183

Karlický, M. 1998, A\&A, 338, 1084

Karlický, M., Chen, B., Gary, D. E., Kašparová, J., \& Rybák, J. 2020a, ApJ, 889, 72

Karlický, M., Kašparová, J., \& Sych, R. 2020b, ApJ, 888, 18

Karlický, M., \& Kliem, B. 2010, SoPh, 266, 71

Karlický, M., \& Odstrčil, D. 1994, SoPh, 155, 171

Karlický, M., \& Rybák, J. 2017, SoPh, 292, 1

Karlický, M., Rybák, J., \& Monstein, C. 2017, SoPh, 292, 94

Karlický, M., Yan, Y., Fu, Q., et al. 2001, A\&A, 369, 1104

Kliem, B., Karlický, M., \& Benz, A. O. 2000, A\&A, 360, 715

Kliem, B., Linton, M. G., Török, T., \& Karlický, M. 2010, SoPh, 266, 91

Krueger, A. 1979, Introduction to solar radio astronomy and radio physics, D. Reidel Publ. Comp., Dordrecht, Holland, p. 115

Lemen, J. R., Title, A. M., Akin, D. J., et al. 2012, SoPh, 275, 17
Li, D., Kolotkov, D. Y., Nakariakov, V. M., Lu, L., \& Ning, Z. J. 2020, ApJ, 888,53

Liu, W., Title, A. M., Zhao, J., et al. 2011, ApJL, 736, L13

Lysenko, A. L., Anfinogentov, S. A., Svinkin, D. S., Frederiks, D. D., \& Fleishman, G. D. 2019, ApJ, 877, 145

Mann, G., Jansen, F., MacDowall, R. J., Kaiser, M. L., \& Stone, R. G. 1999, A\&A, 348, 614

Maxwell, A., \& Thompson, A. R. 1962, ApJ, 135, 138

McLean, D. J., \& Labrum, N. R. 1985, Solar radiophysics : studies of emission from the sun at metre wavelengths, Cambridge University Press,Cambridge, London, p. 55 and 387

Melrose, D. B. 1980, Plasma astrophysics: Nonthermal processes in diffuse magnetized plasmas. Volume 2 Astrophysical applications, Gordon and Breach Science Publ., New York, p. 177

Mohan, A., Mondal, S., Oberoi, D., \& Lonsdale, C. J. 2019, ApJ, 875, 98

Monstein, C. 2016, Journal of the Southeastern Association for Research in Astronomy, 11, 34

Nakariakov, V. M., Foullon, C., Verwichte, E., \& Young, N. P. 2006, A\&A, 452, 343

Nakariakov, V. M., \& Melnikov, V. F. 2009, SSRv, 149, 119

Nakariakov, V. M., Pilipenko, V., Heilig, B., et al. 2016, SSRv, 200, 75

Neupert, W. M. 1968, ApJL, 153, L59

Newkirk, Jr., G. 1961, ApJ, 133, 983

Nishizuka, N., Karlický, M., Janvier, M., \& Bárta, M. 2015, ApJ, 799, 126

Palmer, I. D. 1974, SoPh, 37, 443

Pick, M. 1986, SoPh, 104, 19

Pick, M., Démoulin, P., Krucker, S., Maland raki, O., \& Maia, D. 2005, ApJ, 625, 1019

Poduval, B., DeForest, C. E., Schmelz, J. T., \& Pathak, S. 2013, ApJ, 765, 144

Sakai, J.-I., \& de Jager, C. 1996, SSRv, 77,1

Sharykin, I. N., \& Kosovichev, A. G. 2018, ApJ, 864, 86 
Spicer, D. S., Benz, A. O., \& Huba, J. D. 1982, A\&A, 105, 221

Torrence, C., \& Compo, G. P. 1998, Bulletin of the American

Meteorological Society, 79, 61
Yan, X. L., Wang, J. C., Pan, G. M., et al. 2018, ApJ, 856, 79

Zheleznyakov, V. V., \& Zlotnik, E. Y. 1975, SoPh, 44, 461 


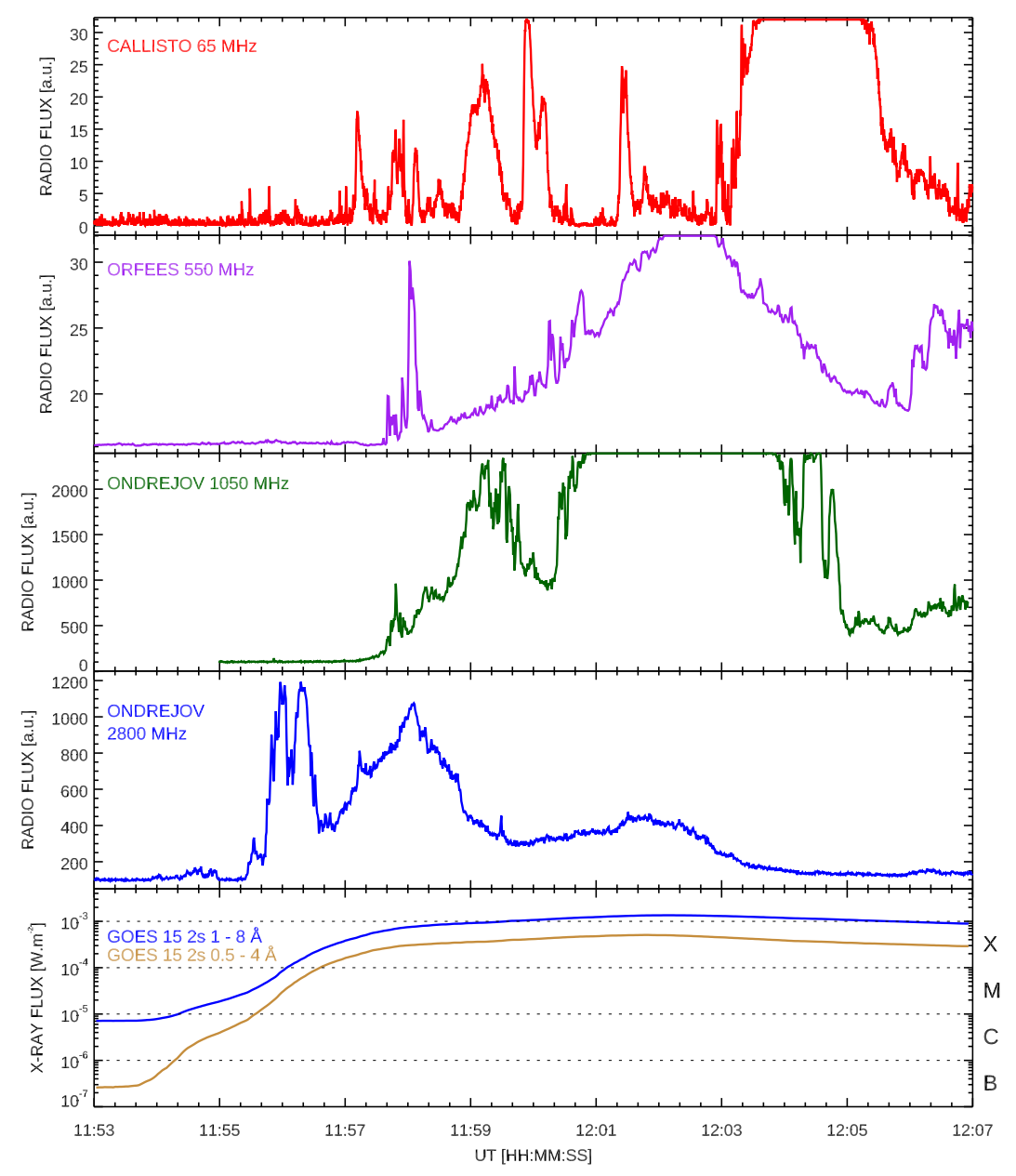

Figure 1. Time profiles of the radio flux on 65, 550, 1050, $2800 \mathrm{MHz}$ and GOES 15 0.5-4 $\AA$ and 1-8 $\AA$ X-ray fluxes during the 2017 September 6 flare.

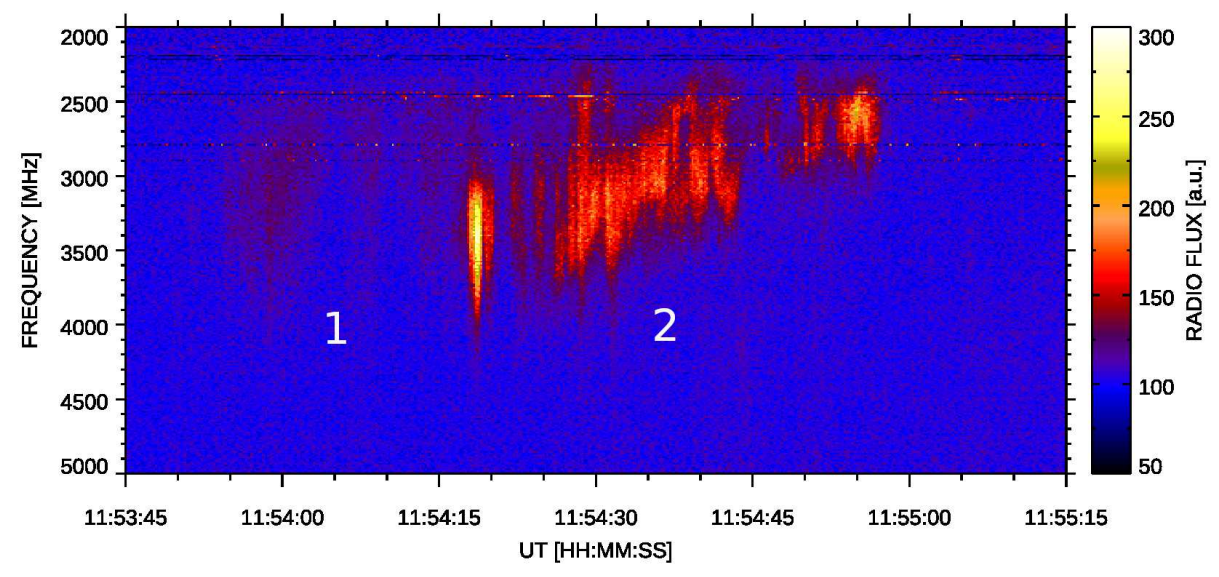

Figure 2. The 2000-5000 MHz radio spectrum showing the double DPS with the parts 1 and 2 in the pre-impulsive flare phase. 

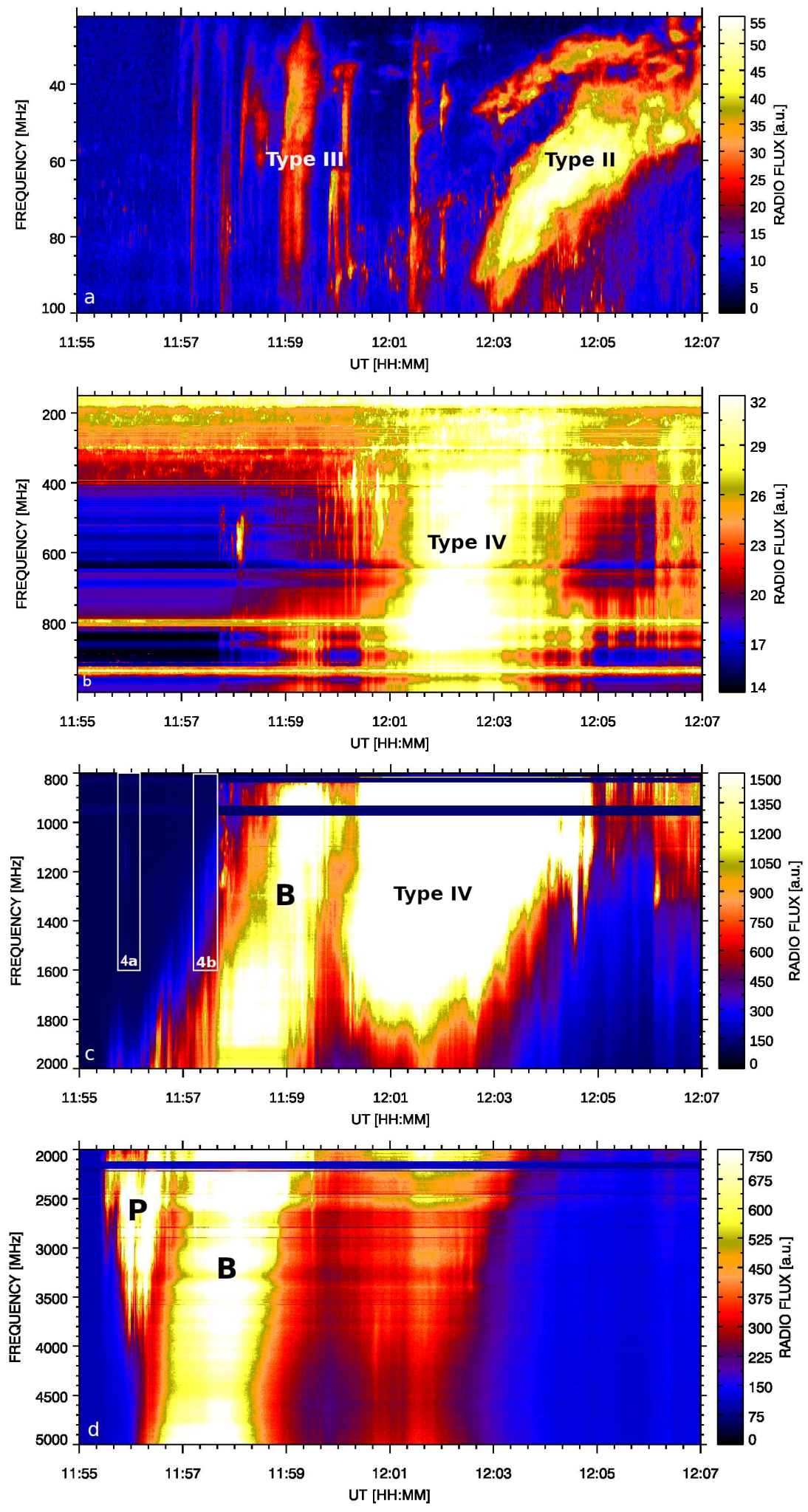

Figure 3. The 22-5000 MHz radio spectrum of the 2017 September 6 flare. a) The 22$100 \mathrm{MHz}$ Greenland-Callisto spectrum, b) the 150-1000 MHz ORFEES spectrum, c) the $800-2000 \mathrm{MHz}$ Ondřejov spectrum, and d) the $2000-5000 \mathrm{MHz}$ Ondřejov spectrum. $\mathrm{P}$ is pulsations and $\mathrm{B}$ means the unusual burst drifting from $5000 \mathrm{MHz}$ to $800 \mathrm{MHz}$ associated with the type III bursts and followed by the type IV and type II bursts. Boxes designated as $4 \mathrm{a}$ and $4 \mathrm{~b}$ in the $800-2000 \mathrm{MHz}$ spectrum show the time-frequency regions, where the detailed spectra, presented in Figures $4 \mathrm{a}$ and $4 \mathrm{~b}$, are shown. 

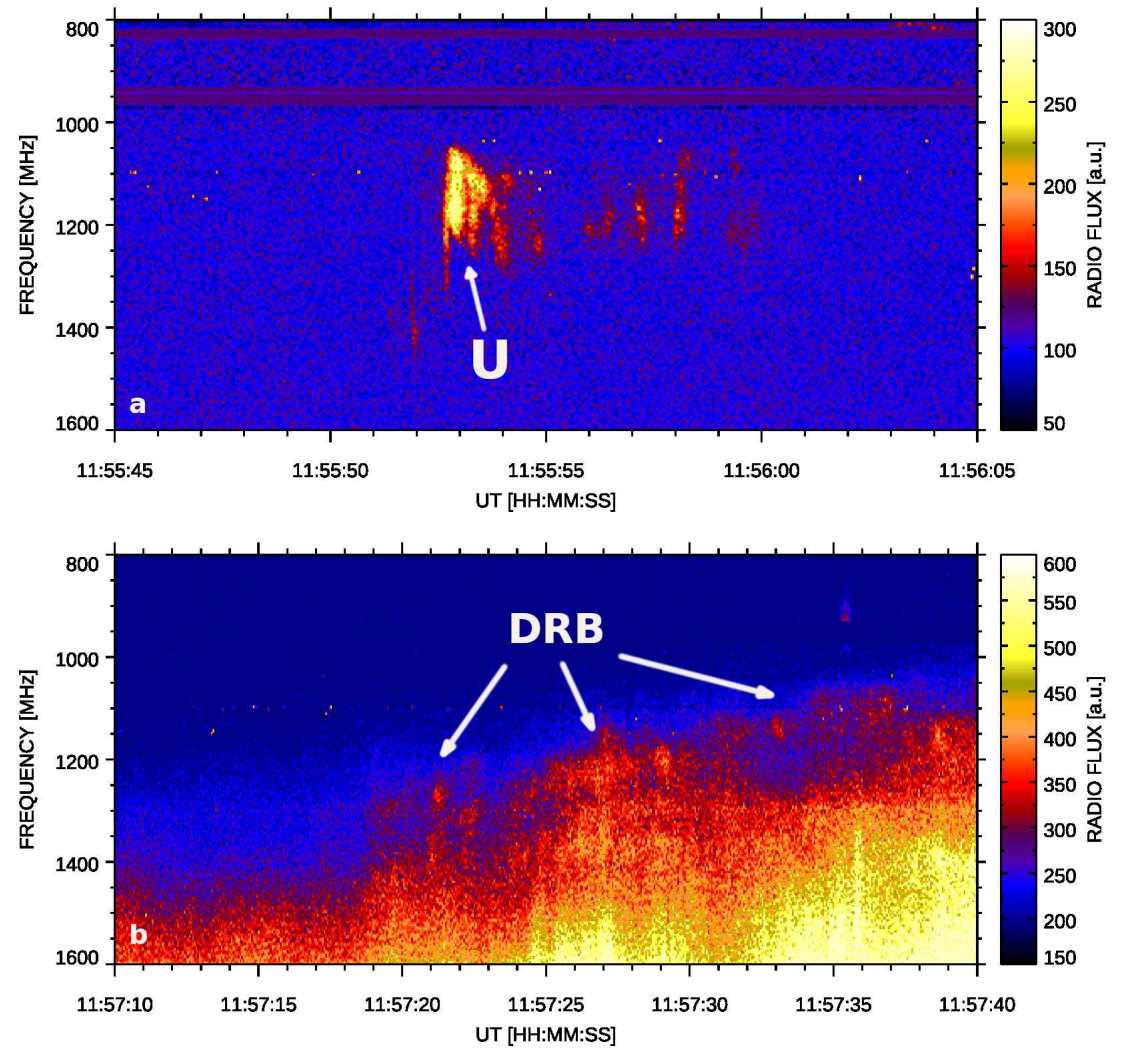

Figure 4. Details of the $800-2000 \mathrm{MHz}$ radio spectrum: a) Group of bursts starting with the high frequency U-burst at 11:55:52-11:56:00 UT in the 1050-1300 MHz range (U), and b) drifting radio burst (DRB) at 11:57:19-11:57:37 UT in the 1050-1350 MHz range close to the low-frequency boundary of the burst B. see also the boxes $4 \mathrm{a}$ and $4 \mathrm{~b}$ in Figure 3. 

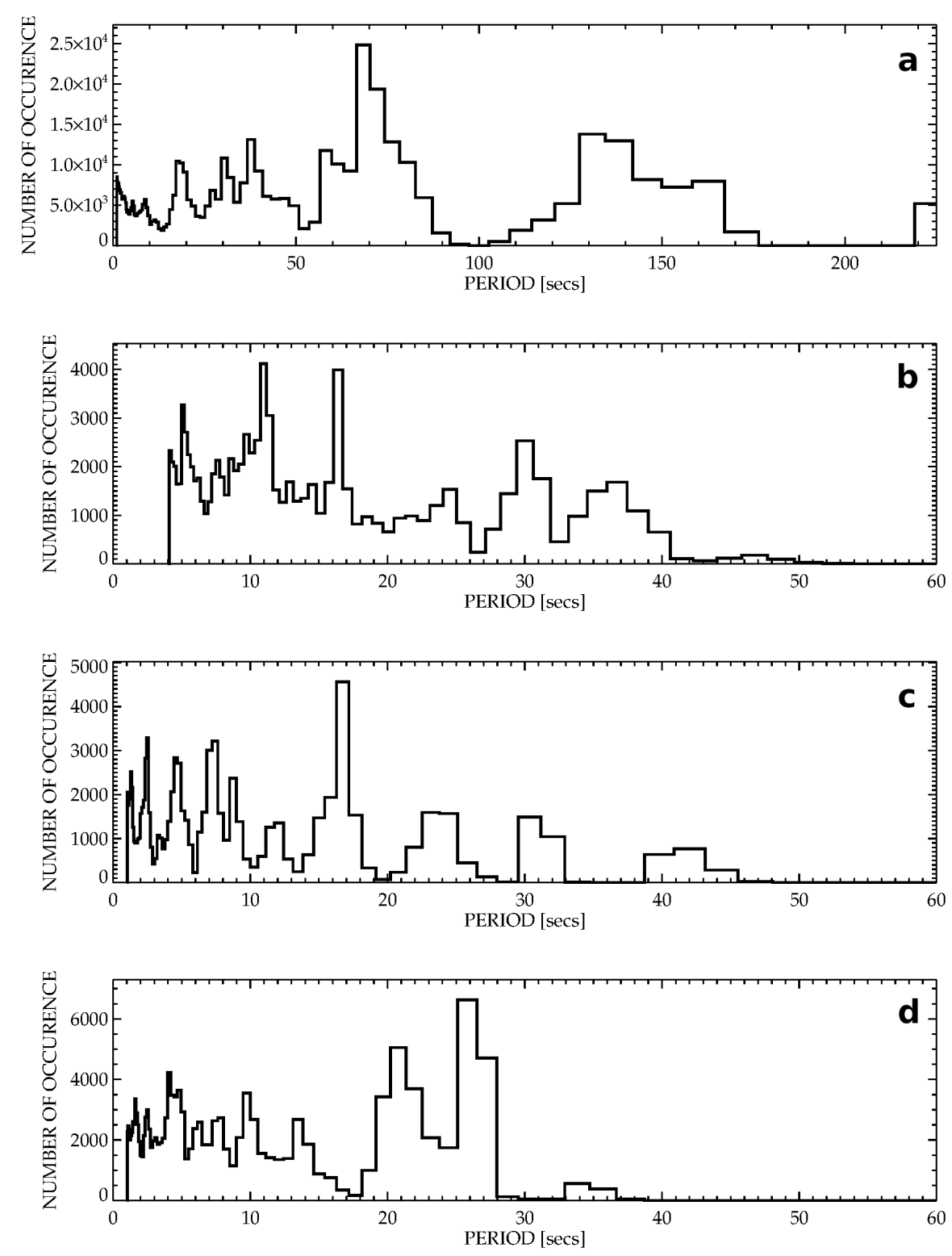

Figure 5. Histograms of periods in the radio spectra of Callisto-Greenland $(22-100 \mathrm{MHz})$ (a), ORFEES (150-1000 MHz) (b), Ondřejov (800-2000 MHz) (c), and Ondřejov (2000$5000 \mathrm{MHz})(\mathrm{d})$. 

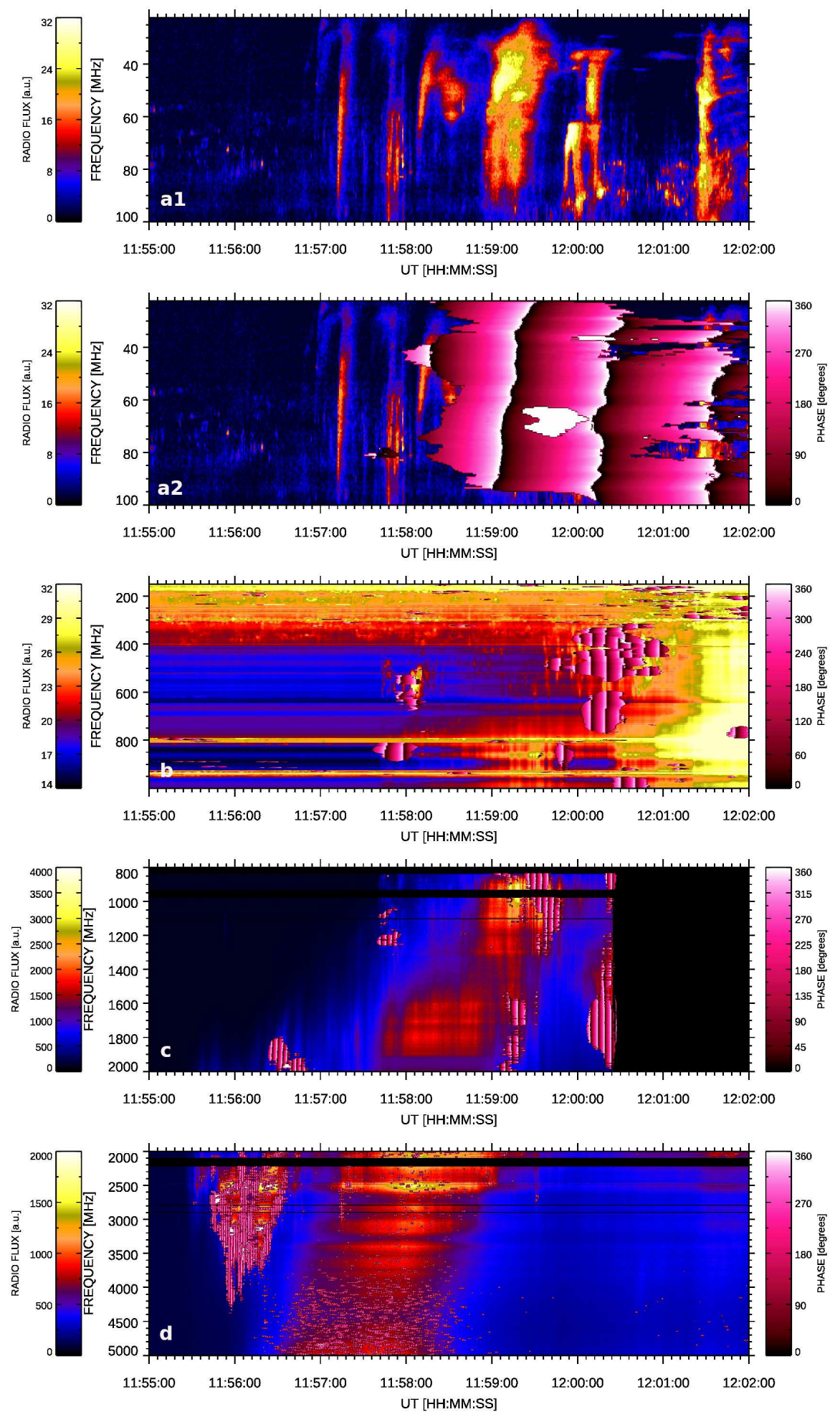

Figure 6. a1) The original 22-100 MHz spectrum. a2)-d) Phase maps (pink areas with the black lines showing the zero phase of pulsations) overplotted on the radio spectrum for periods $53-100 \mathrm{~s}$ in the $22-100 \mathrm{MHz}$ range (a2), 8-14 s in the $150-1000 \mathrm{MHz}$ range (b), 3-6 $\mathrm{s}$ in the $800-2000 \mathrm{MHz}$ range (c), and $1-2 \mathrm{~s}$ in the $2000-5000 \mathrm{MHz}$ range (d). The black part in the 800-2000 MHz spectrum after 12:00:25 UT means saturated radio data. 

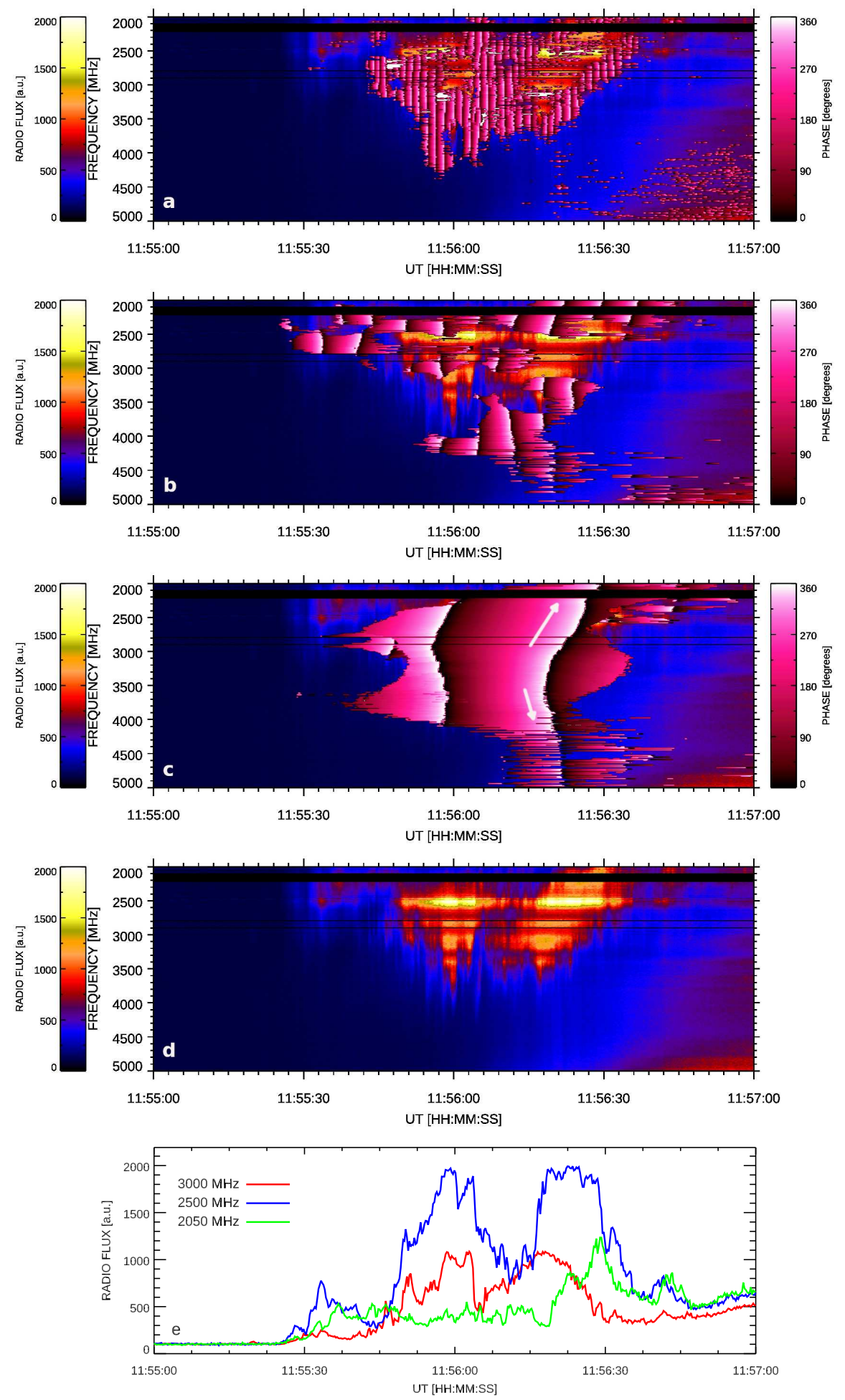

Figure 7. Detailed phase maps of pulsations in the $2000-5000 \mathrm{MHz}$ range, at time 11:5511:57 UT, i.e., at time of the first the $\gamma$-ray peak, for periods $1-2 \mathrm{~s}(\mathrm{a}), 5.3-8.5 \mathrm{~s}$ (b), and 11-30 s (c). Arrows in c) show the bi-directional drift of the pulsation phase. d) The original 2000-5000 $\mathrm{MHz}$ range spectrum. e) The corresponding time profiles of the radio flux on 2050, 2500 and $3000 \mathrm{MHz}$. 


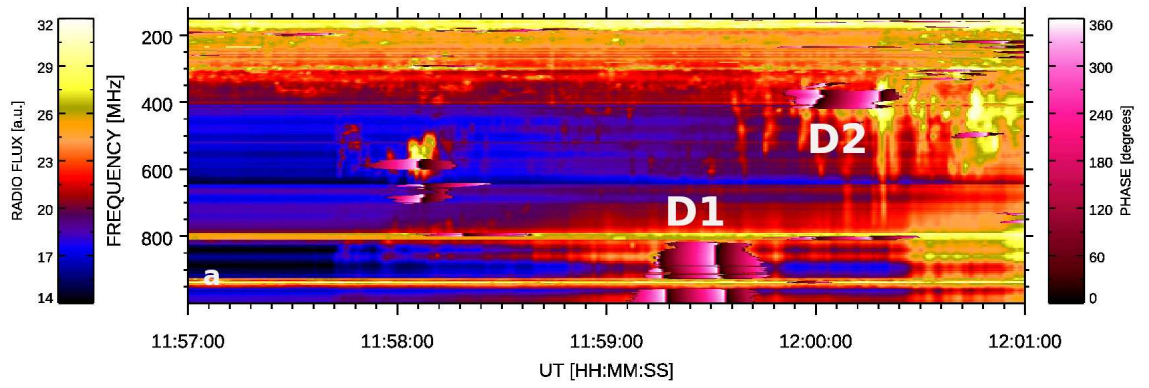

Figure 8. Detailed phase map of pulsations at time 11:57-12:01 UT in the 150-1000 $\mathrm{MHz}$ range for periods $15-20 \mathrm{~s}$ superposed on the original ORFEES spectrum. D1 and D2 designate the pulsations with the phase drifting to higher and lower frequencies, respectively. 

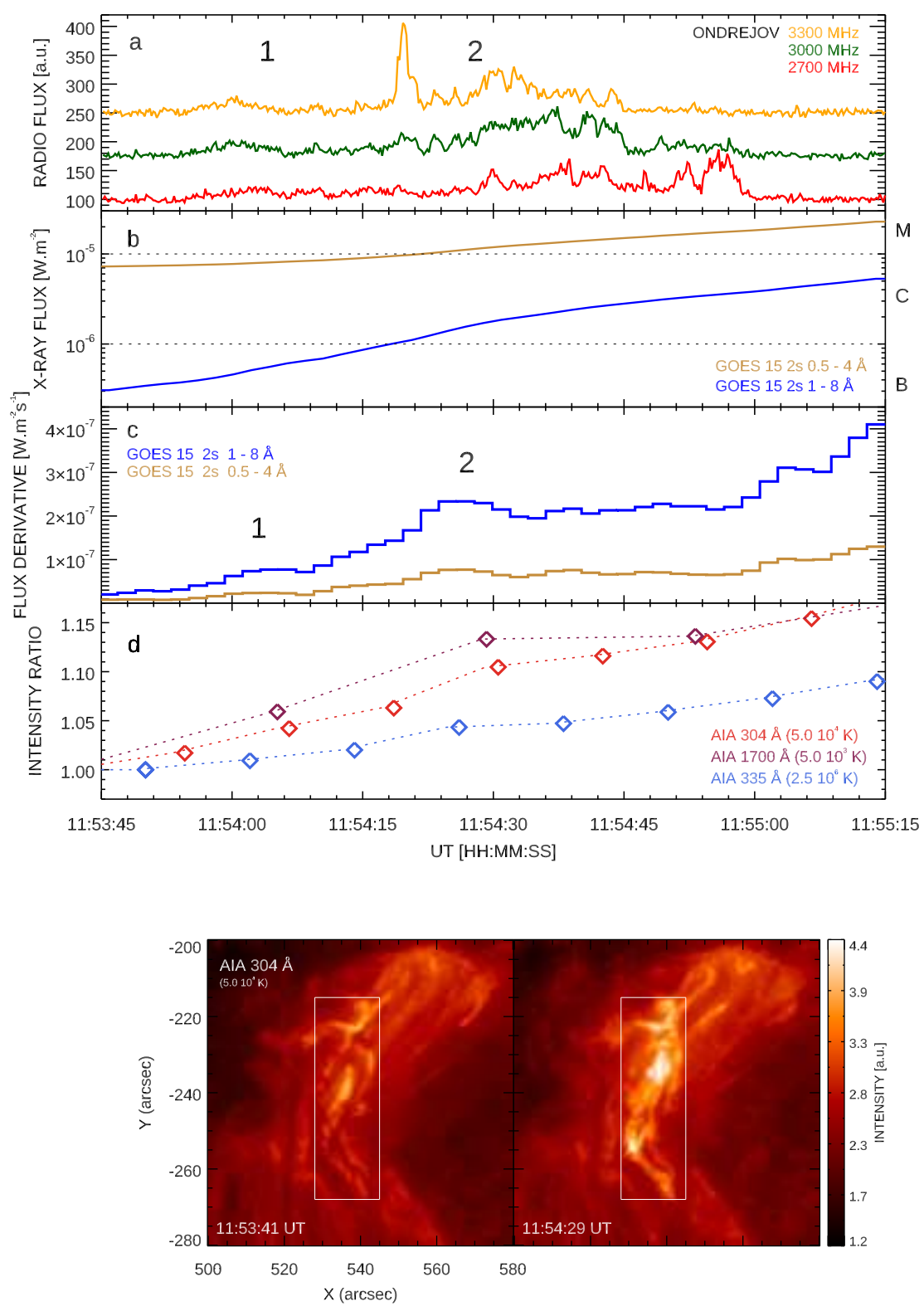

Figure 9. Time profiles of the radio flux on 2700, 3000, $3300 \mathrm{MHz}$ (frequency cuts of DPS) (a), GOES 15 0.5-4 $\AA$ and 1-8 $\AA$ fluxes (b), the time derivative of the GOES 15 fluxes (c), and the AIA 304, 335 and $1700 \AA$ (d) fluxes during the DPS observation. Numbers 1 and 2 in the panel a) mean the part 1 and part 2 of the DPS as designated in Figure 2. Numbers 1 and 2 in the panel c) mean the first weak and second stronger enhancement. Bottom panel: The AIA $304 \AA$ images at times 11:53:41 and 11:54:29 UT with the box showing the region where the AIA fluxes were calculated. 